\title{
The muscular dystrophies
}

\author{
Victor Dubowitz
}

Department of Paediatrics and Neonatal Medicine, Royal Postgraduate Medical School, Hammersmith
Hospital, London W12 ONN, UK

\section{Introduction}

In 1879 William Gowers, the eminent British neurologist, painted a remarkably lucid word picture of Duchenne muscular dystrophy in his series of lectures on pseudohypertrophic muscular paralysis, published in the Lancet. ${ }^{1}$ This disease, he said, is one of the most interesting, and at the same time most sad, of all those with which we have to deal; interesting because of its peculiar features and mysterious nature; sad on account of our powerlessness to influence its course.

Almost exactly a century later Duchenne dystrophy found itself at the centre of one of the most exciting breakthroughs in the modern science of molecular genetics. It was the first genetic disorder in which a previously unknown biochemical abnormality was resolved by the process of reversed genetics, with initial location, isolation and cloning of the gene and then identifying the protein it encodes. Moreover, we now seem to be on the verge of treating the disease by one or other of the potential routes of gene therapy. This would certainly have delighted Gowers. At the same time molecular genetics has generated a most complex and almost totally incomprehensible new jargon of its own, which must surely have turned Gowers in his grave.

The muscular dystrophies, a term first coined by Erb in 1891, are a group of genetically determined disorders characterized by degeneration of skeletal muscle and no associated structural abnormality in the central or peripheral nervous system. They have been subdivided into various clinical types on the basis of the clinical distribution and severity of muscle weakness, and the mode of inheritance. The rate of progression of the disease is also variable and some, such as congenital muscular dystrophy, may remain relatively static or even show functional improvement over time. Some of the animal models of the dystrophies may be devoid of any clinical weakness as may some of the milder variants of, for example, Becker dystrophy, which can present solely with cramps on exercise. ${ }^{1,2}$

Correspondence: Professor V. Dubowitz, M.D., Ph.D., F.R.C.P., D.C.H.
The main breakthrough has been in Duchenne and Becker muscular dystrophy, or the Xp21 dystrophies as some would now have us call them. The location of the gene for the $\mathrm{X}$-linked Emery-Dreifuss muscular dystrophy on the long arm of the $\mathrm{X}$ chromosome at Xq28 was discovered a few years back ${ }^{3}$ but there has as yet been no further progress in its resolution. The locus for the dominantly inherited facioscapulohumeral dystrophy on the long arm of chromosome 4 has only recently been found ${ }^{4}$ and other dystrophies such as the autosomal recessive congenital dystrophy and limb girdle dystrophy will undoubtedly follow suit once sufficient collaborative clinical and laboratory effort is concentrated on them.

\section{The Duchenne dystrophy story}

\section{Locating the gene}

The discovery of the gene locus for Duchenne dystrophy at Xp21 came, almost simultaneously, from three sources. Through the application of the new techniques of molecular genetics it became possible to isolate DNA sequences from cloned fragments derived from the human $\mathrm{X}$ chromosome and to determine their exact location. Duchenne dystrophy was the first disease to be localized in this way by linkage with these restriction fragment length polymorphisms (RFLPs). ${ }^{5}$ Secondly, a number of documented females with Duchenne dystrophy associated with an X:autosomal translocation all had the breakpoint on the $\mathrm{X}$ chromosome at this locus. Thirdly, a number of cases of Duchenne dystrophy with associated Xlinked disorders, such as chronic granulomatous disease, retinitis pigmentosa and McLeod's syndrome were found to have a cytogenetically visible deletion at the same site. ${ }^{6}$ Linkage studies in cases of Becker dystrophy pointed to the same locus, confirming that the two conditions are allelic and due to abnormalities in the same gene. This fits in with the clinical observation that Becker dystrophy has a similar clinical pattern to Duchenne apart from its later onset and milder course and that there is an overlap of cases between the two. ${ }^{1}$ 


\section{The clinical phenotype}

Although it can be argued that all cases of muscular dystrophy associated with the Xp21 gene form a continuous clinical spectrum of a single disease, it would be helpful to have some degree of international consensus on the definition of the different grades of severity of $\mathrm{Xp} 21$ disease, in order to at least be able to compare data from one laboratory with those from another. It is of course also important from the point of view of the patient and his family to be able to give some form of prognosis and appropriate supportive therapy.

The vast majority of boys with Duchenne muscular dystrophy lose the ability to walk independently by the age of 12 years. If one defines Becker dystrophy as maintaining independent ambulation beyond the 16th birthday, this clearly separates the two phenotypes and also recognizes cases of intermediate severity which bridge the gap between the two and lose ambulation between 13 and 16 years. One can then dispense with ill-defined terms such as 'outliers' or 'mild Duchenne' or 'severe Becker'. There is of course considerable variability within the Becker group with some cases remaining ambulant and practically free of disability into late adult life and others having considerable disability already in their late teens. Similarly within the Duchenne group some cases may already be unable to walk by 6 or 7 years of age.

\section{Isolating, cloning and characterizing the gene}

The isolation of the gene was achieved by Kunkel and his colleagues by an ingenious approach of preparing a library of cloned sequences corresponding to the DNA deleted in a patient with Duchenne dystrophy, chronic granulomatous disease, retinitis pigmentosa and McLeod's syndrome and a visible deletion in the $\mathrm{Xp} 21$ region. ${ }^{7}$ This was followed within a comparatively short period of time by the complete cloning of the gene, ${ }^{8}$ and the discovery of the protein product of the gene, which they named dystrophin. ${ }^{9}$ The gene is a gigantic size, encompassing over 2,000 kilobases ( 2 million base pairs), with over 60 exons spanning the gene. Dystrophin is a correspondingly large protein of over 400 kilodalton in size, but with very low abundance, comprising only about $0.002 \%$ of total striated muscle protein.

\section{Clinical applications}

\section{Gene deletions}

Kunkel's group have produced, and made available to genetic laboratories worldwide, a series of cDNA probes covering the whole dystrophin gene. This enables one (in a blood sample) to detect exon deletions within the gene, which occur in about $50-60 \%$ of all Duchenne as well as Becker cases. This has considerably increased the diagnostic potential in carrier detection and antenatal diagnosis (with chorionic villus biopsy in early pregnancy), and also the accurate diagnosis of isolated cases particularly of Becker dystrophy which may have been misdiagnosed as spinal muscular atrophy on the misinterpretation of atrophic fibres in the muscle biopsy, or as autosomal limb girdle dystrophy. It has also helped to confirm the diagnosis of Becker dystrophy in cases presenting with cramps on exercise, a common and well-recognized presenting symptom. ${ }^{1}$ Some of these cases have no detectable weakness or disability but have a grossly elevated creatine kinase and a dystrophic muscle biopsy.

Contrary to early speculations, neither the size of the deletion nor the location bears any consistent relation to the severity of the clinical condition. In a review of 218 of our patients with Duchenne, Becker or intermediate phenotypes, 124 had deletions with the cDNA probes. ${ }^{10}$ Seventy-four separate deletions were found,with 55 being unique to one patient and the remaining 19 occurring in at least two unrelated patients. Some deletions such as of exons 33-34 and 33-35 occurred only with Becker patients and of exons 3-7 in four patients with intermediate severity and one Becker. We also found no correlation of associated mental retardation with any selective deletions.

In order to explain the varying clinical severity, Monaco et al. ${ }^{11}$ postulated that in Duchenne dystrophy the deletion, irrespective of size, leads to a frame shift of the triplet codons for amino acids resulting in a severely truncated non-functional protein, whereas in Becker dystrophy the nucleotides remain in frame, and can produce a functional protein, although reduced in size. Over $90 \%$ of cases with deletions conform to this hypothesis. There have, however, been some exceptions. Thus Malhotra et al. ${ }^{12}$ demonstrated a 3-7 exon deletion, with disruption of the translational frame, in six Becker, five intermediate and two Duchenne cases, and postulated mechanisms that might compensate for the effects of the frame shift in the milder cases.

\section{Dystrophin in muscle biopsies}

In their initial biochemical studies of dystrophin in relation to Duchenne and Becker muscular dystrophies and other neuromuscular disorders, Hoffman et al. ${ }^{13}$ concluded that dystrophin was absent in severe Duchenne dystrophy, was present but of abnormal molecular size in mild Becker muscular dystrophy, and was present in normal 
amounts and size in other forms of muscular dystrophy. It was thus a valuable tool for excluding Duchenne/Becker muscular dystrophy in the case of other forms of dystrophy such as congenital muscular dystrophy or autosomal limb girdle dystrophy that might have a similar clinical presentation. Hoffman also claimed that one might be able to prognosticate in Duchenne or Becker dystrophy on the basis of the dystrophin in the muscle. It almost looked as if the clinician had become redundant and all that was needed was a competent surgeon to take the muscle biopsy and a wellinformed biochemist, working in the splendid isolation of his laboratory, to do the rest! However, not all their cases fitted into this neat compartmentalization. Thus one case of Becker dystrophy had absent dystrophin, which they ascribed to a deletion corresponding to the same DNA region as the fusion proteins used in preparing the original antibodies. By extrapolation this would imply that one would need to know the deletion status of the gene, based on the DNA studies of the patient's blood, before one could draw any conclusions in relation to prognosis from the absence of dystrophin, particulary in a clinically milder case. Conversely, although there was little or no significant dystrophin present in 35 of their 38 cases of Duchenne dystrophy, there were three samples which had detectable levels above $3 \%$ of normal. Similarly in Becker muscular dystrophy the suggestion of altered size or molecular weight of dystrophin excluded the possibility of a normal-sized protein of reduced amount.

The application of immunocytochemical techniques to the study of dystrophin in muscle sections added a new dimension to the interpretation. In contrast to the early suggestion based on the biochemical fractionation studies that dystrophin might be localized to the triads, the cytochemical studies showed a clear-cut localization to the sarcolemmal membrane. ${ }^{14,15}$

While confirming the general absence of any staining for dystrophin in Duchenne biopsies, it has also consistently revealed small numbers, usually less than $1 \%$, of positive staining fibres in some Duchenne biopsies. It has been suggested that these may represent a somatic mutation, with reversion to normal, in individual fibres. In Becker biopsies there may be a variation in the intensity of stain in the positive fibres and also the presence of patchy rather than continuous staining of the membrane in individual fibres. In female carriers the results are also interesting, but somewhat complex. Although manifesting carriers may show a mosaic pattern of positive and negative fibres, ${ }^{16}$ the majority of non-manifesting carriers that we have reviewed have shown an essentially normal dystrophin pattern with practically all fibres showing a normal staining pattern. ${ }^{17}$
In addition to the original Hoffman antibodies, a number of laboratories have produced antibodies to different parts of the dystrophin molecule which has allowed a more comprehensive appraisal of the changes in dystrophin in the muscle in parallel with the deletion studies in the gene. Nicholson et al. ${ }^{16}$ recently reported their experience in a comparative study of immunoblotting and immunocytochemistry in a large series of 226 muscle biopsies, including 85 Duchenne and 55 Becker dystrophies, using a monoclonal antibody to the rod domain of dystrophin, which was specific for dystrophin and did not crossreact with spectrin or alpha-actinin. They found isolated positive fibres in $40 \%$ of the Duchenne biopsies and a further $20 \%$ had weak labelling of a large number of fibres. Of the 54 Duchenne and 52 Becker biopsies showing a positive band on immunolabelling, $85 \%$ had a protein of abnormal size, whereas the remaining $15 \%$ had a protein of normal size but reduced abundance. They found that overall there was a good correlation between the abundance of dystrophin and clinical severity.

A lot more cumulative experience is still required comparing the severity of the clinical disease with the deletion data in relation to the gene and the changes in the dystrophin in the muscle. Meanwhile it would be prudent (for biochemists as well as physicians) not to lose sight of the importance of careful clinical assessment of the patient in relation both to the extent of weakness and the rate of progression of the disease, rather than trying to predict these from the laboratory data in isolation.

\section{Animal models of X-linked (Xp21) dystrophies}

A number of animal models of the Xp21 dystrophy have been identified in recent years and have already attracted a lot of research interest both from the investigative and from the therapeutic point of view.

\section{The mdx mouse}

An X-linked dystrophy in the mouse was picked up purely by chance during a mutagenesis screen of serum enzymes. ${ }^{18}$ Following a period of necrosis of the muscle at about 2-3 weeks of age there is active regeneration and recovery and the mice then remain essentially normal and have a normal lifespan. ${ }^{19}$ The gene for the mouse dystrophy is homologous to the human dystrophin gene, ${ }^{20}$ and dystrophin is also absent in the mdx mouse muscle. ${ }^{9}$ It has recently been shown that the genetic abnormality is a point mutation at nucleotide position 3185 , with the replacement of the nucleotide cytosine by a thymine, resulting in a termination codon (TAA) in place of a glutamine codon (CAA). ${ }^{21}$ This results in premature termina- 
tion of translation at $27 \%$ of the length of the dystrophin polypeptide and the production of a truncated protein. Presumably this residual protein is sufficiently stable and functional to sustain regeneration and fibre integrity in the mouse dystrophy. Possibly some of the human Duchenne and Becker dystrophies cases that do not have a detectable deletion in the gene may have a similar change in a single base pair among the more than 2 million base pairs that make up the gene.

\section{The XMD dog}

An X-linked muscular dystrophy (XMD) has been discovered in a Golden Retriever strain of dogs which is also genetically homologous to the human disease and lacks dystrophin in the muscle. ${ }^{22,23}$ Clinically the dog manifests a severe weakness with an early onset and steady progression, comparable to the Duchenne type. From an early stage there is also marked wasting of the muscles, but none of the prominence and apparent enlargement of the muscles one sees in the human disease. The histological picture is identical to that in Duchenne dystrophy, including the early proliferation of endomysial connective tissue. Selective involvement of some muscles at an earlier stage than others has been demonstrated in the neonatal period, ${ }^{24}$ comparable to the selective involvement from an early stage in Duchenne dystrophy.

The exact genetic mutation has not yet been identified, but no deletions have been found. As in the case of the mdx mouse, all the descendants within the same strain will presumably have the same mutation. Cooper has recently diagnosed a dystrophin-deficient dystrophy in a pair of sibling male Rottweiler pups, aged 5 months ${ }^{25}$ Clinically they were more severely affected than the previous Retriever progeny. The canine X-linked muscular dystrophy is thus likely to affect many different strains of dog, and a number of case reports of myopathies in dogs in the earlier veterinary literature may well have been cases of abnormalities in the dystrophin gene.

\section{The XMD cat}

A dystrophin-deficient muscular dystrophy has been documented in a single neutered male cat, which was not clinically weak and had marked prominence of the muscles. ${ }^{26}$ Cooper has recently investigated a similar male cat with a dystrophindeficient dystrophy, which had a remarkable degree of muscular hypertrophy and no obvious clinical weakness. This feline model of muscular dystrophy is of special interest in parallel with the mouse dystrophy for its absence of clinical weakness and its marked muscle hypertrophy, which probably also occurs in the mdx mouse, and is, of course, also a feature of selected muscles in the human disease, the so-called pseudohypertrophy already noted by Duchenne, who coined the term.

\section{Therapeutic possibilities}

\section{Gene therapy}

The dramatic advances in the past few years in relation to the dystrophy gene naturally raised the hopes of sufferers and their families that a cure might be around the corner. It also sparked speculation in the scientific community on the possibility of gene therapy. Techniques already exist for the introduction of DNA into somatic or germline cells, either directly or with viral or other vectors. One major hurdle was the tremendous size of the gene which would be beyond the capacity of retroviral vectors. Possible ways around this were the use of alternative vectors such as yeast artificial chromosomes or the construction of a 'mini' gene from the DNA of a patient with mild Becker type dystrophy and the deletion of about half of the dystrophin, yet still capable of producing a functional protein about half the normal size. ${ }^{27}$

Moreover it has recently been shown that DNA injected directly into muscle remains functionally viable, thus bypassing the need for a vector. ${ }^{28}$ To crown it all, Caskey's laboratory have just succeeded in producing a construct of the whole dystrophin gene, and shown that the recombinant dystrophin that it produced was indistinguishable from mouse muscle dystrophin by Western blot analysis with anti-dystrophin antibodies, and was localized to the cell membrane with fluorescent antibody techniques. ${ }^{29}$ This adds further credence and hope to the possibilities of direct gene therapy, but there are still major hurdles to be overcome such as the targeting of the replacement gene to the affected muscles.

\section{Myoblast transfer}

An attractive, feasible and potentially practical short cut, which could provide an alternative approach to the replacement of either the gene or its protein product, has been the introduction of normal muscle cells, in the form of actively dividing cultured myoblasts, directly into the dystrophic muscle. These myoblasts can then fuse with the regenerating mononucleate satellite cells of the host, which lie dormant under the sarcolemmal membrane of normal as well as dystrophic muscle, until activated. The multinucleate muscle cells formed are thus a combination of normal and dystrophic cells, comparable to the female carrier of the Duchenne gene, and hopefully sufficiently functional to provide reasonable muscle power. 
Earlier experimental work in mice showed the fusion of donor and host myoblasts to produce hybrid multinucleate muscle cells, ${ }^{30}$ and to introduce genes from the donor cells. ${ }^{31}$ This approach was subsequently used by Law and his colleagues ${ }^{32}$ to try to correct the abnormality in the autosomal recessive muscular dystrophy in the mouse. In a recent application of the technique in the $\mathrm{mdx}$ mouse, Partridge and his colleagues demonstrated the fusion of normal donor muscle cells with the muscle cells of the host with the production of hybrid multinucleate fibres. These fibres were also capable of expressing dystrophin, which was consistently absent in the host muscle. ${ }^{33}$

While the mdx mouse is a good model for establishing the experimental aspects of this therapeutic approach, and has the advantage of the small muscle size in relation to the number of injected cells, it is not a good model for assessing potential clinical benefit in the absence of any clinical weakness. In this respect the XMD dog, with its clinical phenotype and pathological picture more closely resembling Duchenne dystrophy, should provide a better model.

\section{Human myoblast transfer experiments}

Fired by the apparent success of the technique in the mdx mouse, a number of centres in North America opted to plunge forthwith into experiments in patients with Duchenne dystrophy to evaluate the potential therapeutic value in the context of the human disease. They anticipated that they would be able to resolve such basic questions as the minimum and optimal number of cells needed to be effective, the method of introduction and dispersion of the cells into the host muscle, and the short-term or long-term need for immunosuppression, as part of the experimental design. As in any therapeutic trial, it is absolutely essential to have objective and reliable methods to assess the outcome of the experiment, both from the point of view of the successful fusion of the donor with the host cells and the expression of dystrophin in a previously dystrophin-negative subject, and also the effect this has on the pathological state of the muscle, and its clinical function.

From an immunological compatibility point of view, the ideal donor would probably be a sibling, but in view of the ethical constraints on using minors in the context of such experiments, the father has usually been chosen.

Karpati and his team in Montreal, Canada, are injecting cultured myoblasts from the father into the biceps muscle of boys with proven Duchenne dystrophy and an associated deletion in the dystrophin gene. This is done randomly and blindly, the other biceps having a comparable injection without the myoblasts in. The presence of dystrophin in the injected muscles on both sides is being assessed after 3 months in open biopsy samples with both Western blotting and immunocytochemical techniques. The muscle strength in the elbow flexors is also being measured sequentially.

Law and his colleagues in Memphis, USA, are conducting a similar series of experiments on the extensor digitorum brevis muscle on the dorsum of the foot, using cultured myoblasts from the father, and comparing the results with the sham-injected opposite side. The small size of the muscle has potential advantages in relation to the number of cells needed for the procedure, but limitations if more than one biopsy were envisaged or in accurate measurement of muscle force. These studies are still very much in the experimental stage. It will not be feasible to proceed to the next phase of actual therapeutic trials of myoblast transfer in appropriate patients to assess the potential impact on the disease itself until we have a clear-cut and unequivocal answer to the basic question as to whether the technique produces significant and adequate expression of dystrophin in the host muscle, and what beneficial effect this has on the muscle pathology and particularly on the clinical function. It is also imperative for the wide publicity that this work has already had in the lay press to be toned down in order to avoid unwarranted hope in the sufferers and their families together with the frustration of thinking (misguidedly) that they are already missing out on actual beneficial treatment by not being where the action lies.

Meanwhile, we may be able to get a further appraisal of the therapeutic potential of this technique in the dystrophic dog, and I have established a collaborative programme with Dr Barry Cooper's group at the New York State College of Veterinary Medicine at Cornell University in Ithaca, USA. There are a number of advantages in the dog model. One can use siblings as donors and carefully control the timing of the injections in relation to the stage of the pathological process and also compare different muscles which may be at different stages in the same animal. There are also major hurdles to be overcome in the canine model such as the difficulty in culturing cloned myoblasts in quantity with existing culture media which may be optimal for human or mouse muscle, and the relatively rudimentary status of knowledge in relation to the dog's immunological systems, compared to man or mouse.

\section{Conclusions}

The muscular dystrophies are currently at a very exciting stage of active resolution of many of the fundamental questions that have puzzled and 
eluded clinicians and scientists for the past century. The Duchenne gene has been cloned, its protein identified and its location accurately mapped to the inner surface of the sarcolemmal membrane. Its function has not yet been fully established but it is thought to have a stabilizing role in the integrity of the membrane in close association with linked glycoproteins. The absence or abnormality of dystrophin presumably triggers the process of progressive degeneration and disintegration of the muscle cell, but the other factors, such as the influx of calcium, which have an important role in perpetuating the process still await more detailed study and clarification.

The new era of potential treatment of the remorselessly progressive and disabling Duchenne form of muscular dystrophy is just beginning, and things look set fair for its success, given the remarkable technical advances we have witnessed in relation to gene manipulation in the last few years.

\section{Dedication}

I am delighted to contribute this paper on the muscular dystrophies to the festschrift for Lord Walton and to express my personal appreciation for all the help and advice he has given me in so many ways over the years, since my interest in muscular dystrophy was first kindled during a residency post at Queen Mary's Hospital for Children in 1957 and also my gratitude to the Muscular Dystrophy Group of Great Britain for their continuous support of my research endeavours over the past 33 years.

\section{References}

1. Dubowitz, V. Muscle Disorders in Childhood. W.B. Saunders, London, Philadelphia, Toronto, 1978.

2. Dubowitz, V. A Colour Atlas of Muscle Disorders in Childhood. Wolfe Medical Publications, London/Year Book Publishers, Chicago, 1989.

3. Hodgson, S., Boswinkel, E., Cole. et al. A linkage study of Emery-Dreifuss muscular dystrophy. Hum Genet 1986, 74: 409-416.

4. Wijmenga, C., Frants, R.R., Brouwer, O.F., Moerer, P., Weber, J.L. \& Padberg, G.W. Location of facioscapulohumeral muscular dystrophy gene on chromosome 4 . Lancet 1990, 336: 651-653.

5. Murray, J.M., Davies, K.E., Harper, P.S. et al. Linkage relationship of a cloned DNA sequence on the short arm of the X-chromosome to Duchenne muscular dystrophy. Nature 1982, 300: 69-71.

6. Francke, U., Ochs, H.D., de Martinville, B. et al. Minor Xp21 chromosome deletion in a male associated with expression of Duchenne muscular dystrophy, chronic granulomatous disease, retinitis pigmentosa and McLeod syndrome. Am J Hum Genet 1985, 37: 250-267.

7. Kunkel, L.M., Monaco, A.P., Middlesworth, W. et al. Specific cloning of DNA of a male patient with an Xchromosome deletion. Proc Natl Acad Sci USA 1985, 82: $4778-4782$.

8. Koenig, M., Hoffmann, E.P., Bertelson, C.K. et al. Complete cloning of the Duchenne muscular dystrophy (DMD) cDNA and preliminary genomic organisation of the DMD gene in mouse and affected individuals. Cell 1987, 50: 509-517.

9. Hoffman, E.P., Brown, R.H. \& Kunkel, L.M. Dystrophin: The protein product of the Duchenne muscular dystrophy locus. Cell 1987, 51: 919-928.

10. Hodgson, S.V., Hart, K.H., Abbs, S. et al. Correlation of clinical and deletion data in Duchenne and Becker muscular dystrophy. J Med Genet 1989, 26: 682-693.

11. Monaco, A.P., Bertelson, C.J., Liechti-Fallati, S. et al. An explanation for the phenotypic differences between patients bearing partial deletions of the DMD locus. Genomics 1989 , 2: $90-95$.

12. Malhotra, S.B., Hart, K.A., Klamut, H.J. et al. Frame-shift deletions in patients with Duchenne and Becker muscular dystrophy. Science 1988, 242: 755-759.

13. Hoffman, E.P., Fishbeck, K.H., Brown, R.H. et al. Characterization of dystrophin in muscle-biopsy specimens from patients with Duchenne's or Becker's muscular dystrophy. $N$ Engl J Med 1988, 318: 1363-1368.
14. Zubrzycka-Gaarn, E.E., Bulman, D.E., Karpati, G. et al. The Duchenne muscular dystrophy gene product is localised in the sarcolemma of human skeletal muscle. Nature 1988, 333: 466-469.

15. Arahata, K., Ishiura, S., Ishiquro, T. et al. Immunostaining of skeletal and cardiac muscle surface membrane with antibody against Duchenne muscular dystrophy peptide. Nature 1988, 333: 861-863.

16. Nicholson, L.V.B., Johnson, M.A., Gardner-Medwin, D., Bhattacharya, S. \& Harris, J.B. Heterogeneity of dystrophin expression in patients with Duchenne and Becker muscular dystrophy. Acta Neuropathol Berl 1990, 80: 239-250.

17. Clerk, A., Rodillo, E., Heckmatt, J.Z., Dubowitz, V., Strong, P.N. \& Sewry, C.A. Characterization of dystrophin in carriers of Duchenne muscular dystrophy. J Neurol Sci (in press).

18. Bulfield, G., Siller, W.G., Wight, P.A.L. \& Moore, K.J. X chromosome-linked muscular dystrophy $(\mathrm{mdx})$ in the mouse. Proc Natl Acad Sci USA 1984, 81: 1189-1192.

19. Dangain, J. \& Vrbova, G. Muscle development in $\mathrm{mdx}$ mutant mice. Muscle Nerve 1984, 7: 700-704.

20. Hoffman, E.P., Monaco, A.P., Feener, C.C. \& Kunkel, L.M Conservation of the Duchenne muscular dystrophy gene in mice and humans. Science 1987, 238: 347-350.

21. Sicinski, P., Geng, Y., Ryder-Cook, A.S. et al. The molecular basis of muscular dystrophy in the mdx mouse: A point mutation. Science 1989, 244: 1578-1580.

22. Valentine, B.A., Cooper, B.J., Cummings, J.F. \& de Lahunta, A. Progressive muscular dystrophy in a golden retriever dog: Light microscope and ultrastructural features at 4 and 8 months. Acta Neuropathol 1986, 7: 301-310.

23. Cooper, B.J., Winand, N.J., Stedman, H. et al. The homologue of the Duchenne locus is defective in X-linked muscular dystrophy of dogs. Nature 1988, 334: 154-156.

24. Valentine, B.A. \& Cooper, B.J. Canine X-linked muscular dystrophy: Selective involvement of muscles in neonatal dogs. Neuromusc Disord 1991, 1: 31-38.

25. Cooper, B.J. Personal communication.

26. Carpenter, J.L., Hoffman, E.P., Romanul, F.C. et al. Feline muscular dystrophy with dystrophin deficiency. Am J Pathol 1989, 135: 909-919.

27. England, S., Nicholson, L.V.B., Johnson, M.A. et al. Very mild muscular dystrophy associated with the deletion of $46 \%$ of dystrophin. Nature 1990, 343: 180-182. 
28. Wolff, J.A., Malone, R.W., Williams, P. et al. Direct gene transfer into mouse muscle in vivo. Science 1990, 247: $1465-1468$.

29. Lee, C.C., Pearlman, J.A., Chamberlain, J.S. \& Caskey, C.T. Expression of recombinant dystrophin and its localization to the cell membrane. Nature 1991, 349: 334-336.

30. Partridge, T.A., Grounds, M. \& Sloper, J.C. Evidence of fusion between host and donor myoblasts in skeletal muscle grafts. Nature 1978, 273: 306-308.

31. Watt, D.J., Morgan, J.E. \& Partridge, T.A. Use of mononuclear precursor cells to insert allogeneic genes into growing mouse muscles. Muscle Nerve 1984, 7: 741-750.
32. Law, P.K., Goodwin, T.G. \& Wang, M.G. Normal myoblast injections provide genetic treatment for murine dystrophy. Muscle Nerve 1988, 11: 525-533.

33. Partridge, T.A., Morgan, J.E., Coulton, G.R. et al. Conversion of mdx myofibres from dystrophin-negative to -positive by injection of normal myoblasts. Nature 1989, 337: 176-179. 